\title{
CUMLAUDE
}

CUM LAUDE

Revista del Doctorado en Derecho

Facultad de Derecho y Ciencias Sociales y Políticas UNNE

$\mathrm{N}^{\circ} 2$ - Septiembre 2015

Corrientes - Argentina

ISSN: 2422-6408

info@revistacumlaude.com

FECHA DE RECEPCIÓN: 15/05/2014

FECHA DE ACEPTACIÓN: 25/03/2015

ARbitraRiedad EN LAS SENTENCIAS DE LOS TRIBUNALES DE CHACO

Y CORRIENTES: UN ANÁLISIS DE CASOS DESDE LA MIRADA DE CHAÏM

Perelman y Robert Alexy

Sergio López Pereyra

Facultad de Derecho, Ciencias Sociales y Políticas

UNIVERSIDAD NACIONAL DEL NORDESTE

RESUMEN

Arbitrariedad en las Sentencias de los tribunales de Chaco Y Corrientes: un anÁlisis de casos desde la mirada de ChaÏ̀ Perelman y ROBERT ALEXY

Este trabajo tiene como objeto colaborar, a través de la lectura y el análisis de jurisprudencia de los Superiores Tribunales de Justicia de las provincias de Chaco y Corrientes, con el estudio acerca de la arbitrariedad y sus causales en el derecho. Se ha 


\section{ARBITRARIEDAD EN LAS SENTENCIAS DE LOS TRIBUNALES DE CHACO Y CORRIENTES: \\ UN ANÁlisis DE CASOS DESDE LA MIRADA DE CHAÏM PERELMAN Y ROBERT ALEXY}

decidido a los fines del análisis, realizar el trabajo tomando como modelo cuatro fallos de los Superiores Tribunales de Justicia de las provincias de Chaco y Corrientes. Nos valdremos en particular de los aportes de las teorías de Robert Alexy y Chaïm Perelman, teóricos de la argumentación jurídica, y de precedentes de la Corte Suprema de la Nación. El trabajo pretende acercar al lector una guía que intenta establecer patrones judiciales seguidos para determinar cuándo se encuentra ante decisiones judiciales arbitrarias para cuestionarlas judicialmente.

\section{Palabras claves}

Causales de arbitrariedad; teorías de la argumentación jurídica; falta de fundamentación; jurisprudencia Nordeste; sentencia arbitraria.

\section{Abstract}

ARbITRARINESS IN SENTENCES OF CHACO AND CORRIENTES COURTS: AN ANALYSIS OF CASES FROM THE THEORY OF CHAIM PERELMAN AND ROBERT AleXY

This work aims to contribute, through the reading and analysis of jurisprudence of the High Courts of Chaco and Corrientes, with the studies about arbitrariness and its causes in the law. With theoretical basis in the argumentation theory and the analysis of the jurisprudence of the Superior Courts of the Provinces of Corrientes and Chaco, this study pretends to bring to the reader a guide that tries to establish judicial patterns to determine when sentences are lacking of fundamentation. We will use in particular the contributions of the theories of Robert Alexy and Chaim Perelman and precedents of the Supreme Court of the Nation. 


\section{KEYWORDS}

Arbitrariness - theory of legal argumentation - sentences - northeast -jurisprudence.

\section{INTRODUCCIÓN}

Este trabajo tiene como objeto colaborar, a través de la lectura y el análisis de jurisprudencia de los Superiores Tribunales de Justicia de las provincias de Chaco y Corrientes, con el estudio acerca de la falta de fundamentación y sus causales en el derecho.

Se ha decidido a los fines del análisis, realizar el trabajo tomando como modelo cuatro fallos: Sentencia No 63 del 03/08/09 del S.T.J. de Corrientes, "Brizolla, Juan y Binni de Brizzolla Ramona Ester s/sucesorio"; Sentencia No 126 del 08/09/08del S.T.J. de Corrientes, "Bachini Patricia Silvana s/homicidio culposo-Santa Lucía"; Sentencia No 315 del 03/09/08del S.T.J. del Chaco, “Orellana, Epifania c/Diego Gabriel Polic s/daños y perjuicios y/o Josefa Merka vda. de Polic s/daños y perjuicios" y Sentencia del 21/07/06 del S.T.J. del Chaco “Aranda Miguel Florentino, Vallejos Raúl Andrés s/Homicidio simple en concurso real con homicidio en grado de tentativa reiterada en concurso real"

Debemos mencionar que se utilizan aquí todos los elementos y herramientas obtenidas en el desarrollo del PI $\mathrm{N}^{\circ}$ 105/08, acreditado por Secretaría General de Ciencia y Técnica de la Universidad Nacional del Nordeste. ${ }^{1}$

\footnotetext{
${ }^{1} \mathrm{PI} \mathrm{N}^{\mathrm{o}} 105$ "Fundamentación y arbitrariedad en las sentencias de los Superiores Tribunales de Justicia de las provincias del nordeste: su análisis comparativo desde la teoría de la argumentación jurídica”.
} 


\section{ARBITRARIEDAD EN LAS SENTENCIAS DE LOS TRIBUNALES DE CHACO Y CORRIENTES: UN ANÁlisis DE CASOS DESDE LA MIRADA DE CHAÏM PERELMAN Y ROBERT ALEXY}

Nos valdremos en particular de los aportes de las teorías de Robert Alexy (1989) y Chaïm Perelman (1989), teóricos de la argumentación jurídica y de precedentes de la Corte Suprema de la Nación, con el objeto de poder identificar cuándo los Superiores Tribunales de Justicia locales entienden que se está en presencia de una sentencia arbitraria por falta de fundamentación.

\section{Síntesis DE LAS PROPUESTAS QUE INTERESAN DE LA TEORÍA DE LA}

\section{ARGUMENTACIÓN JURÍDICA}

Nuestro trabajo tiene como objeto de estudio la falta de fundamentación en las sentencias de los Superiores Tribunales de las Provincias de Chaco y Corrientes que han sido enumerados en la introducción. Como se afirma que los estudios sobre los recaudos para fundar las decisiones judiciales en nuestro país son escasos, aquí, buscaremos abordar algunos criterios sentados por la Corte Suprema de Justicia de la Nación y su repercusión en la Corte Local.

Así, teniendo en cuenta las herramientas que nos brinda las teorías de Alexy y Perelman, realizaremos un análisis de las sentencias escogidas, que corresponden a los Superiores Tribunales de Justicia de las provincias mencionadas.

En este sentido hemos de procurar anticipar una tendencia, (aunque $\sin$ pretensiones estadísticas) en la línea de razonamiento y preferencias argumentativas de nuestros magistrados a la hora de fundar sus decisiones, todo esto con la finalidad de vincularlo al propósito medular del trabajo.

De esta manera, podemos intentar generalizar de los casos examinados, en forma similar a la obtención de los objetos de acuerdos en Perelman, una línea de 
coincidencias en orden a los argumentos y razones vertidos en diferentes fallos, para luego sí, señalar cambios, diferencias o variantes axiológicas en todos ellos.

Es interesante destacar a manera de recaudo, que tanto en los argumentos (considerandos) de los fallos evaluados se omiten referencias doctrinarias concretas a los autores propuestos o a menciones de obras en teorías de argumentación.

También resulta evidente que la utilización de ciertos argumentos, criterios o pautas son empleados convenientemente a la luz de la praxis jurisdiccional y sin invocación expresa o apego a algunas de las doctrinas propiciadas en nuestra indagación.

Es así que rescatamos en los fallos mencionados la realidad, quizás lícita, del uso indiferenciado o en forma de sinonimia de vocablos tales como razón y argumento en aras de la simplicidad, pero que a nuestro juicio el primero de ellos implica un reconocimiento a la verdad más allá de nuestros pareceres, en tanto que el argumento (más allá de su bondad) muchas veces carece de razón, diferencias estas que exceden las de una simple cuestión semántica, dado que podríamos emitir conclusiones basadas en buenas argumentaciones, un auditórium podría aceptar conclusiones basadas únicamente en buenos argumentos, basta con que el argumento de las conclusiones descansen en premisas con argumentos similares.

Por otra parte, en los fundamentos leídos se advierte la coincidencia en dar preponderancia a las circunstancias envolventes a los hechos, siendo esta apreciación relevante dado que no siempre se expresan en forma literal de qué manera se elaboran las premisas que más tarde fundarán el decisorio judicial, esto es así por que como se sabe, los hechos controvertidos en un proceso judicial son casi siempre anteriores al mismo, por lo que su comprobación empírica en orden a su ocurrencia cabal es casi 


\section{ARBITRARIEDAD EN LAS SENTENCIAS DE LOS TRIBUNALES DE CHACO Y CORRIENTES: UN ANÁLISIS DE CASOS DESDE LA MIRADA DE CHAÏM PERELMAN Y ROBERT ALEXY}

imposible, de tal manera que las premisas que surjan de esos hechos deberán ser buscadas o construidas con diferentes argumentos que funcionarán como pruebas o presunciones y como tales tendrán carácter conjetural y falible.

Ahora bien, dentro de esta precaria construcción empírica de hechos ocurridos con anterioridad a su ponderación nuestros juzgadores elaboran sus premisas que operarán como puntos de inducción para su futura y definitiva valoración, por lo que el juicio corre peligro de ser un poco menos que objetivo al quedar siempre supeditadas esas generalizaciones a la esperanza de su eficacia o aceptación por ser un buen argumento. Esta realidad admitida por parte de nuestros funcionarios, con o sin referencias a teorías o doctrinas, implica el interrogante de si nuestros fallos transitan del deber ser al ser, de la razón al argumento.

Ello así, con el agregado de que cada sujeto es poseedor de un argumento, distinto al de los otros con relación a un mismo hecho, dependiendo en último análisis del poder de persuasión del argumento mismo con relación al sujeto y a la suposición de éste acerca de la probabilidad de que, esos mismos argumentos tengan alguna eficacia de convencer a los demás. O en palabras de Alexy (1989) al afirmar que, para que un argumento sea bueno o plausible, no es suficiente que un tribunal diga que la gente en la realidad usa tales argumentos, sino que además un número suficiente de personas acepte, por lo menos a largo plazo, esos argumentos como razones de corrección. Solo las personas racionales son capaces de aceptar argumentos sobre la base de su corrección o sensatez.

La admisión de estas premisas que dan partida a diferentes argumentos constituye sin dudas la base de consideración empírica de nuestros jueces, circunstancia esta que sienta el peligro de que malos argumentos, aunque convincentes, abran las 
puertas a sentencias arbitrarias, o más sutil aún, que una argumentación evidentemente verdadera por corresponder a una proposición descriptiva carezca de atingencia lógica con la conclusión.

Para estas cuestiones Alexy (1989) relativiza el problema haciendo la distinción entre personas razonables y personas irrazonables, en las creencias de las primeras se vuelven objetivas las argumentaciones.

En otros aspectos de la teoría de la argumentación y cotejándola con algunos de los fallos examinados, se hace visible el reconocimiento de nuestros magistrados al hacer prevalecer decisiones que sean asimiladas por la sociedad en forma justa $\mathrm{y}$ razonable, así por ejemplo el máximo tribunal de la provincia de Corrientes lo entiende en un caso donde se revoca la sentencia de una cámara de apelaciones por entender que es posible llegar a una decisión razonable y aceptable sin desconocer la sistemática jurídica como derivaciones lógicas. En este sentido las postulaciones de Chaïm Perelman (1989) al sostener la posibilidad de persuadir dentro de una lógica jurídica material, no formal, admiten la motivación inherente a toda decisión judicial con el fin de convencer a todo el auditorio, afirmando que las decisiones judiciales descansan sobre juicios de valor como las decisiones morales o cualquier otro discurso de carácter axiológico. De esta manera el resultado de la argumentación será racional cuando se considere valido para un auditorio idealmente constituido por el conjunto de todos los espíritus razonables. En el fallo observado entendemos que el razonamiento jurídico se detuvo en una simple subsunción silogística, en la mera apariencia, se avocó a un razonamiento técnico y no ético por lo que el máximo tribunal con buen sentido concluye el análisis con una interpretación racional que integra una solución asimilada por la sociedad en forma justa y racional con el respeto necesario al derecho. 


\section{ARBITRARIEDAD EN LAS SENTENCIAS DE LOS TRIBUNALES DE CHACO Y CORRIENTES: UN ANÁLISIS DE CASOS DESDE LA MIRADA DE CHAÏM PERELMAN Y ROBERT ALEXY}

En otras palabras, lo que el Máximo Tribunal de Justicia de la Provincia de Corrientes deja traslucir en los autos "Brizzolla, Juan y Binni de Brizzolla, Ramona Ester s/sucesorio",2, y aunque no lo diga expresamente en el proceso argumental, es que en el mundo de los valores no pueden predicarse verdades absolutas y eternas, siendo posible justificar racionalmente las opciones valorativas que se ofrecen en los sujetos aunque no aparezcan en términos de verdades absolutas como en el mundo de lonatural, es esta lógica valorativa la que merece ser llamada de "razonabilidad" en opinión de Perelman, solo así es posible justificar racionalmente una decisión jurídica, siendo por otra parte este control de racionalidad una misión permanente a cargo de los jueces, haciéndose claro que la razonabilidad de las decisiones no aparecen de manera evidente sino que debe ser justificada retóricamente, entendido esto como el uso de los medios que permiten convencer, argumentar y acrecentar la adhesión de las voluntades a la tesis (sentencia) que se someten a su asentimiento.

En otra línea de análisis dentro de la moderna teoría de la argumentación jurídica y con relación a un fallo de Superior Tribunal de Justicia de la Provincia de Corrientes ${ }^{3}$ es pasible advertir con nitidez aspectos de la dogmática jurídica planteada en Alexy al hacerse visible en la argumentación del fallo algunas de las funciones positivas que hacen al principio de justicia.

En efecto, la sentencia analizada pone sobre relieve (aunque nuevamente sin mención de seguimiento doctrinario) la función de control de consistencia al fundar enunciados dogmáticos para hacer prevalecer la garantía de juez imparcial, frente a una decisión de tribunales colegiados inferiores, tribunal este que había receptado un recurso

\footnotetext{
${ }^{2}$ S.T.J. de Corrientes, Sentencia No $63,03 / 08 / 2009$.

${ }^{3}$ S.T.J. de Corrientes, Sentencia No 126 del 08/09/08 "Bachini Patricia Silvana s/homicidio culposo-Santa Lucía”.
} 
de casación sobre un caso donde los mismos magistrados ya habían resuelto lo que ahora se intentaba casar, realizando la Cámara de Apelaciones en lo Criminal una doble actuación para un caso concreto, a lo que el Superior Tribunal de la Provincia de Corrientes entendió en una apreciación dogmática y analógica que debía recepcionarse un planteo de nulidad ejercido por el defensor del imputado y por el Sr. Fiscal General a efectos de salvaguardar la garantía de imparcialidad de los jueces aludida.

También podemos hallar y considerar en nuestras indagaciones otras pautas de la argumentación jurídica, siguiendo directivas del pensamiento de Robert Alexy (1989) en lo que hace a las funciones de estabilización y progreso, estas circunstancias se advierten sin inconvenientes en el razonamiento de magistrados del Superior Tribunal de la provincia de $\mathrm{Chaco}^{4}$ en una resolución que dictamina sobre un Recurso Extraordinario por Arbitrariedad de sentencia deducido por la parte vencida en los autos “Orellana, Epifania c/ Diego Gabriel Polic s/ Daños y Perjuicios”, actuaciones estas en la que los jueces del máximo tribunal de esa provincia hacen ver las funciones que cumple la dogmática jurídica, como estabilización observable en la tarea de compatibilización lógica de los enunciados dogmáticos entre si, orientados permanentemente con menciones doctrinarias. Estas funciones se justifican porque permiten a los magistrados que junto a los enunciados dogmáticos se fijen determinadas soluciones prácticas permitiendo que en determinados periodos de tiempo se tomen formas de decisión similares, afianzándose el principio de universalidad, sin que ello implique que un enunciado dogmático deba ser mantenido por tiempo ilimitado, admitiéndose con esto la función de progreso vinculada a la anterior que permite aceptar los cambios de ideas valorativas dentro de la sociedad y en los legisladores.

\footnotetext{
${ }^{4}$ S.T.J. del Chaco, Sentencia No 315 del 03/09/2008.
} 


\section{ARBITRARIEDAD EN LAS SENTENCIAS DE LOS TRIBUNALES DE CHACO Y CORRIENTES: UN ANÁLISIS DE CASOS DESDE LA MIRADA DE CHAÏM PERELMAN Y ROBERT ALEXY}

Podemos trabajar en el análisis de casos, también, con la distinción propuesta por Chaïm Perelman (1989) entre el auditorio universal y el auditorio concreto o particular.

Esta distinción entendemos puede actuar como marco o telón de fondo, a los fines de establecer los argumentos más concretos y vinculados a una moral social predominante en una determinada comunidad de operadores jurídicos; allí estaría presente el Auditorio Concreto, el mensaje de la decisión o sentencia judicial a los fines de vincular la expresión del discurso jurídico, con una serie de razones materiales que operan a los fines de la justificación interna de la decisión del juez o tribunal. Por otra parte nos encontramos con la necesidad de estilizar los argumentos jurídicos para un auditorio universal, en el sentido de que la decisión o sentencia también va dirigida a un auditorio de seres racionales y como modelo de comportamiento o paradigma del modo de resolver los conflictos que tiene una determinada comunidad jurídica que comparte valores y principios con una comunidad no sólo nacional, sino internacional.

En este sentido, podemos acudir a los precedentes sentados por la Corte Suprema de Justicia de la Nación en el caso "Casal". Allí la Corte entiende que el recurso de casación penal, no debe limitarse a ser un remedio extraordinario y limitado, y debe en cambio permitir una revisión amplia en el caso de sentencia condenatoria; así expone que "El Estado nacional argentino tiene la obligación de reformar su legislación procesal penal de modo de sustituir el recurso de casación -extraordinario y limitadopor un recurso ordinario que permita al tribunal superior un examen integral de la decisión recurrible a través del amplio conocimiento de la causa, y cuyo único límite

\footnotetext{
${ }^{5}$ C.S.J.N, "Casal, Matías Eugenio y otro s/ robo simple en grado de tentativa, 20/09/2005.
} 
estaría dado por aquello que surja de manera directa y excluyente de la inmediación, y de cuyos pormenores no existiera constancia adecuada (del voto de la Dra. Highton de Nolasco)". Son los hechos del caso Casal que determinaron el criterio de la Corte de que el recurso extraordinario y limitado de Casación, no cumplía con la Constitución respecto de la incorporación de los Tratados Internacionales de Derecho Humanos, en cuanto determinan la Convención Americana sobre Derechos Humanos Art. $8^{\text {o }}$ Garantías Judiciales, Inc. h) el derecho de recurrir de fallo ante juez o tribunal superior. El criterio sentado por la Corte, por supuesto que también impacta fuertemente como criterio para fallar en los Superiores Tribunales de Justicia Provinciales.

En la provincia del Chaco en el caso "Aranda"6 la Cámara Segunda en lo Criminal de la Ciudad de Resistencia condena a los imputados. Contra la sentencia se alzó únicamente la defensa de uno de ellos, interponiendo recurso de inaplicabilidad de la ley, y así llegó a tratamiento del Superior Tribunal, donde el máximo tribunal local procedió a rechazar la impugnación, denegándose posteriormente la concesión del recurso extraordinario federal interpuesto contra dicho fallo en Febrero de 2005. La Corte Suprema de Justicia, por mayoría, declaró procedente el recurso de queja y dejó sin efecto el pronunciamiento de la Sala Penal del Superior Tribunal de Justicia, devolviendo la causa para que por quien corresponda, se dicte nuevo fallo con arreglo a la doctrina Casal, sentencia de fecha 20 de Septiembre de 2005.

El caso Aranda, es un fallo de transición entre el criterio anterior sobre el recurso de casación, como un recurso limitado y restrictivo, al criterio del caso Casal, donde pasa ser clave la ampliación del recurso de casación, a la manera de un recurso de

\footnotetext{
${ }^{6}$ S.T.J. del Chaco , Sentencia No 91 del 21/07/06 "Aranda Miguel Florentino; Vallejos Raúl Andrés s/Homicidio en simple en concurso real con homicidio en grado de tentativa reiterada en concurso real".
} 


\section{ARBITRARIEDAD EN LAS SENTENCIAS DE LOS TRIBUNALES DE CHACO Y CORRIENTES: UN ANÁLISIS DE CASOS DESDE LA MIRADA DE CHAÏM PERELMAN Y ROBERT ALEXY}

revisión amplio, ya que es la manera de asegurar la garantía constitucional de la doble instancia.

Aplicando las categorías de los distintos auditorios propuestos por Perelman, podríamos decir que el fallo de la Corte Suprema de Justicia de la Nación tiende, mediante los argumentos del Caso Casal, tratar de atender a un hipotético auditorio universal, y la sentencia de la Sala Penal del Superior Tribunal de Justicia del Chaco, atiende básicamente al auditorio particular, más precisamente al conjunto de justiciables de la Provincia del Chaco.

Se establece una visión diferente entre lo que reclama por un lado, la Corte Suprema de la Nación, como un debido esfuerzo de racionalidad y de extensión del recurso de Casación, tendiente a lograr un recuso amplio de revisión a los fines de cumplir con la Constitución en cuanto a los tratados Internacionales de Derechos Humanos, y la posible responsabilidad del Estado ante Tribunales internacionales, lo que constituiría una manifestación del auditorio universal propuesto por Perelman.

Ante una primera impresión podemos decir que estamos en presencia de un argumento (por parte de la Corte Suprema) de tipo "idealista" ya que se propondría como una situación ideal a alcanzar en busca de poner al día, de acuerdo a las normas internacionales hoy constitucionalizadas, y en términos de Perelman, como ya dijimos, de apuntar al auditorio universal.

Pero realizando un análisis del argumento de la Corte podemos sostener que se asoma la posición utilitaria, en el sentido de que se vuelve a hacer referencia a las disposiciones constitucionales que podrían acarrear perjuicios al Estado. La Corte alude a la necesidad de evitar sanciones al Estado Argentino como motivo sustancial para plantear los criterios que deben seguir los Tribunales inferiores, y no en una auténtica

\section{SERGIO LÓPEZ PEREYRA}


exposición de argumentos que apunten a la convicción de la necesidad de que en los fallos condenatorios se debe extremar las medidas y prevenciones a los fines de respetar las garantías constitucionales del imputado. Se basa en un discurso dirigido a ciudadanos racionales, que deberían comprender y participar eventualmente en las críticas al criterio sentado, ya que es un tema que hace a una sociedad organizada, y central a los efectos de diseñar e implementar instituciones que decidan, fallen y propongan argumentos o fundamentos de sus decisiones basados en principios, en convicciones y dirigidas a un auditorio universal. Además el discurso racional práctico especial, que es el jurídico, se desarrolle de manera racional y en una secuencia que busque la persuasión y apunte a la convicción del auditorio, y no sólo a la advertencia de evitar sanciones internacionales.

\section{CONCLUSIONES DEL ANÁLISIS JURISPRUDENCIAL}

La pretensión de seguridad jurídica asociada al derecho objetivo ha generado el mito de certeza del derecho, consistente en suponer que la aplicación de la ley no habrá de producir arbitrariedad. Ese ideal de certeza se asocia con la creencia en la infalibilidad de las decisiones judiciales. Sin embargo, un importante sector de la doctrina advierte sobre el margen de discrecionalidad que los jueces tienen en la aplicación de la norma.

La noción de arbitrariedad surge como un reconocimiento de lo que de hecho ocurre en la praxis de la decisión judicial, aún cuando en la motivación judicial haya referencia a una norma en sentido objetivo. En teoría, la idea de arbitrariedad es rechazada por el derecho, que requiere una fundamentación de todas las acciones; sin embargo el plano de la arbitrariedad existe. 


\section{ARBITRARIEDAD EN LAS SENTENCIAS DE LOS TRIBUNALES DE CHACO Y CORRIENTES: \\ UN ANÁLISIS DE CASOS DESDE LA MIRADA DE CHAÏM PERELMAN Y ROBERT ALEXY}

Como la arbitrariedad es materialmente posible pero filosóficamente inaceptable, en la conciencia jurídica moderna, se nos plantea el problema de encontrar los modos de evitarla.

En la actualidad tenemos el remedio del Recurso Extraordinario Federal para resguardar la constitucionalidad de las decisiones de los magistrados. Tanto por las razones expuestas por el artículo 14 de la Ley 48 como por la creación pretoriana de arbitrariedad, se pueden atacar las sentencias que adolezcan de estas causales.

A través de este remedio legal se custodia los derechos, principios y garantías consagradas en la Carta Magna.

Si bien la Corte Suprema de Justicia de la Nación fue delineando los caracteres, requisitos y causales de esta nueva creación, ella como los Superiores Tribunales de Justicia de las provincias de Chaco y Corrientes, utilizan expresiones que se asocian a la falta de fundamentación pero que no son equivalentes. Hacen referencia a la falta de fundamentación y otras a la falta de fundamentación suficiente. Estas expresiones y sus significados, no siempre se encuentran bien definidas y dada la naturaleza argumentativa de la fundamentación es importante estudiar esta cuestión, con herramientas otorgadas por la teoría de la argumentación.

Sin embargo, los estudios sobre la arbitrariedad y la fundamentación de la sentencia, son teóricos (Habermas, 1989; Alexy, 1989; Perelman, 1989 se han ocupado del tema), y no suponen un estudio de casos sistemáticamente efectuado, al menos en el plano nacional.

Lo novedoso es proponer un estudio de casos a partir del cual construir, desde la herramienta de las respectivas teorías de la argumentación jurídica de los autores citados, los recaudos mínimos de fundamentación de las sentencias judiciales para evitar 
la arbitrariedad; en otras palabras, es necesario desplazar el eje de discusión teórica sobre la arbitrariedad al plano de la construcción de las decisiones judiciales.

Es por ello que para evitar la arbitrariedad en la sentencias, otro de los modos posibles es la elaboración de cánones de fundamentación de sentencias dirigidos a la totalidad del cuerpo del Poder Judicial y de los abogados litigantes, previa sistematización de las exigencias que en cada caso los órganos de máxima jerarquía han establecido como recaudos mínimos de fundamentación.

En lo que concierne al ámbito provincial, tanto de Chaco como de Corrientes existen mecanismos para atacar una sentencia arbitraria. En la provincia de Corrientes nos encontramos con los Recursos de Nulidad, Inaplicabilidad de ley y el de Inconstitucionalidad, que nos permite llegar al Superior Tribunal de Justicia para lograr revocar una sentencia con tacha de arbitrariedad.

En la provincia del Chaco, se encuentra legislado el Recurso Extraordinario Federal y podemos llegar a él a través de los recursos de inconstitucionalidad e inaplicabilidad de ley o doctrina legal.

De acuerdo al análisis de los fallos de los Superiores Tribunales de Justicia, se observa que en el Chaco, se continúa una tradición uniforme en cuanto a consideraciones de causales, terminología derivada de fallos de la Corte Suprema de Justicia y libros de clásicos, en materia de Recurso Extraordinarios. En esta provincia, el máximo tribunal local, sigue los lineamientos y la postura de Corte Suprema así como de autores clásicos en lo que al tema se refiere. De esta manera, los litigantes saben con precisión, cuál es el criterio seguido por el Superior Tribunal chaqueño. Basta con leer los fallos que hemos analizado, para dar cuenta de ello. 


\section{ARBITRARIEDAD EN LAS SENTENCIAS DE LOS TRIBUNALES DE CHACO Y CORRIENTES: UN ANÁLISIS DE CASOS DESDE LA MIRADA DE CHAÏM PERELMAN Y ROBERT ALEXY}

Diferente es lo que sucede en el Superior Tribunal de Justicia de Corrientes donde si bien globalmente se utilizan expresiones jurisprudenciales de la Corte Suprema de Justicia de la Nación, el tribunal local incorpora conceptos distintos, expresiones diferentes, que merecen análisis particular. Por lo cual, sugerimos a los jueces, que tengan en cuenta que si bien el Superior Tribunal de Corrientes, cita a la Corte Suprema de Justicia que establece que se tengan en cuenta la totalidad de las cuestiones conducentes al pleito, el tribunal local hace referencia a una sola cuestión. ${ }^{7}$

Además el máximo tribunal provincial introduce conceptos derivados de la normativa nacional y por ello se exigen aclaraciones. Utiliza conceptos sin establecer su alcance -por ejemplo el de "sana crítica" analizado en el caso "Gómez Lisandro Alberto c/Gabriel Rivero s/daños y perjuicios"-; tampoco podemos obtener estos conceptos de doctrinas jurisprudenciales, debido a su inexistencia. Es por este motivo que debemos remitirnos a diversos autores procesalistas a fin de obtener el significado de estos conceptos, pero con la dificultad de ser estos muy generalizados.

De lo anterior se impone un examen de dos conceptos "madres" que se utilizan en provincia de Corrientes para la falta de fundamentación, absurdo y sana crítica. Al no estar determinado, por parte del superior tribunal, el alcance que se le da a estos conceptos, sugerimos a los litigantes que tengan en cuenta esta situación al momento de recurrir a la justicia por medio de Recurso Extraordinario.

Sugerimos al juez local, los litigantes, fiscales, peritos y todos los agentes del derecho que intervienen en los procesos judiciales, la importancia de plantear un glosario conceptual que evite arbitrariedad. Así como por ejemplo en autos "García,

\footnotetext{
${ }^{7}$ De acuerdo surge del fallo analizado S.T.J de Corrientes, Sentencia No 78 del 27/11/2008 "Rodríguez, Adriano c/Alberto Tránsito Savaresse y/o C.O.O s/reivindicación".
} 
Gustavo M. c/Gladis Godoy y/o q.r.r. s/indemnización” se necesita de precisión con respecto a la vaguedad del concepto de absurdo, por ser una palabra imprecisa, siendo necesario saber que se entiende por ello.

Concluyendo, del análisis de fallos se establecen diferencias cualitativas entre las diferentes jurisdicciones, en tanto la causal de falta de fundamentación de la provincia del Chaco, deriva de la continua utilización de usos y convenciones terminológicas de la Corte Suprema, y ello no ocurre en la provincia de Corrientes. Ésta última, incorpora conceptos propios de una teoría de la interpretación judicial que tiene como base normativa los códigos procesales civiles y penales, lo que obliga un examen particularizado de los conceptos.

\section{REFERENCIAS BIBLIOGRÁFICAS}

Alexy, R. (1989). Teoría de la Argumentación Jurídica., Centro de Estudios Constitucionales: Madrid.

Perelman, Ch. (1989). Tratado de argumentación. La nueva retórica. Traducción española de Muñoz Julia. Sevilla - Madrid: E. Gredis.

S.T.J de la Provincia del Chaco, "Toledo, Antonia Elvira e/a: Mateo Dino, Raimundo José c/Toledo, Elvira y otros/juicio ejecutivo", Sentencia $N^{\circ}$ del 274 del $14 / 08 / 2007$

S.T.J de la Provincia del Chaco, “Zuazquita, Héctor Oscar s/medida cautelar”, Sentencia $\mathrm{N}^{\mathrm{o}} 230$ del $21 / 06 / 27$.

S.T.J. de la Provincia de Corrientes, "Brizolla, Juan y Binni de Brizzolla Ramona Ester s/sucesorio", Sentencia No 63 del 03/08/2009. 
S.T.J. de la Provincia de Corrientes, "Bachini Patricia Silvana s/homicidio culposoSanta Lucía”, Sentencia No 126 del 08/09/2008.

S.T.J. de la Provincia de Corrientes, "Boo, Héctor José s/Sucesión testamentaria", Sentencia No 172 del 12/10/06.

S.T.J. de la Provincia de Corrientes, "Haidar Alberto Ángel Alejandro c/Daniel Hetch s/ordinario (reivindicación, daños y perjuicios) Acumulado Etch, Daniel c/Municipalidad de Yapeyú (Ctes.) y/o quien o quienes se consideren propietarios s/ordinario", Sentencia No 9 del 27/02/08.

S.T.J. de la Provincia de Corrientes, "Rodríguez, Adriano c/Alberto Transito Savaresse y/o C.O.O s/reivindicación”, Sentencia No 78 del 27/11/08.

S.T.J. de la Provincia del Chaco “Aranda Miguel Florentino, Vallejos Raúl Andrés s/Homicidio simple en concurso real con homicidio en grado de tentativa reiterada en concurso real", Sentencia del 21/07/2006.

S.T.J. de la Provincia del Chaco, "Orellana, Epifania c/Diego Gabriel Polic s/daños y perjuicios y/o Josefa Merka vda. de Polic s/daños y perjuicios”, Sentencia No 315 del 03/09/2008.

\section{Curriculum Vitae}

El autor es Abogado, Doctor en Ciencias Jurídicas, Profesor Titular de la Cátedra de Filosofía del Derecho de la Universidad Nacional del Nordeste, Director Titular del Departamento de Formación General, Jurídica, Investigación y Práctica Profesional de la Facultad de Derecho de la UNNE, Profesor Estable del Doctorado en Derecho de la Facultad de Derecho de la UNNE.

Correo electrónico: sergiolopezpereyra@outlook.com 